\title{
Time series behavior modeling with digital twin for Internet of Vehicles
}

\author{
Tianle Zhang ${ }^{1}$, Xiangtao Liu', Zongwei Luo ${ }^{2}$, Fuqiang Dong ${ }^{3}$ and Yu Jiang ${ }^{*^{*}}$ (D)
}

\begin{abstract}
Electric vehicle (EV) is considered eco-friendly with low carbon emission and maintenance costs. Given the current battery and charging technology, driving experience of EVs relies heavily on the availability and reachability of EV charging infrastructure. As the number of charging piles increases, carefully designed arrangement of resources and efficient utilization of the infrastructure is essential to the future development of EV industry. The mobility and distribution of EVs determine the charging demand and the load of power distribution grid. Then, dynamic traffic pattern of numerous interconnected EVs poses great impact on charging plans and charging infrastructure. In this paper, we introduce the digital twin of a real-world EV by modeling the mobility based on a time series behaviors of EVs to evaluate the charging algorithm and pile arrangement policy. The introduced digital twin EV is a virtually simulated equivalence with same traffic behaviors and charging activities as the EV in real world. The behavior and route choice of EVs is dynamically simulated base on the time-varying driving operations, travel intent, and charging plan in a simulated large-scale charging scenario composed of concurrently moving EVs and correspondingly equipped charging piles. Different EV navigation algorithms and charging algorithms of Internet of Vehicle can be exactly evaluated in the dynamic simulation of the digital twins of the moving EVs and charging infrastructure. Then we analyze the collected data such as energy consumption, charging capacity, charging frequency, and waiting time in queue on both the EV side and the charging pile side to evaluate the charging efficiency. The simulation is used to study the relations between the scheduled charging operation of EVs and the deployment of piles. The proposed model helps evaluate and validate the design of the charging recommendation and the deployment plan regarding to the arrangement and distribution of charging piles.
\end{abstract}

Keywords: Electric vehicle, Internet of Vehicles, Mobility, Time series, Digital twin, Charging scheduling

\section{Introduction}

With the advantage of fuel efficiency and greenhouse gas (GHG) reduction, electric vehicles (EVs) have been taking an increasingly greater share in the private and commercial automobile market. Given the current battery technology, charging problem is still the bottleneck of the progress of EV industry. Thus, it is necessary to build and deploy a wide-area charging infrastructure which embraces fast charging piles, battery swapping stations, and individual charging points for slower EV battery charging [1]. The convenience and utilization of charging infrastructure is the key to EV operations. Plug-in EVs are also ideal for fleet managers due to

\footnotetext{
* Correspondence: jiangyu@gzhu.edu.cn

${ }^{1}$ Cyberspace Institute of Advanced Technology, Guangzhou University, Guangzhou 510006, China

Full list of author information is available at the end of the article
}

better energy efficiency, lower cost, and fewer operational resources to maintain and manage. More and more motor vehicle fleets are investing in EVs and planning to convert vehicle fleet to battery powered. Rapid growth in the demand of electricity may bring higher peak demand at the distribution level of the charging grid of EV. While the widespread adoption of EVs brings potential social and economic benefits, the impact of EV charging cannot be overlooked.

The unsuitable deployment and arrangement of charging infrastructure may cause potential transformer overloads, feeder congestion, and undue circuit faults. Carefully designed plan should be made to achieve balanced and robust operation of smart grid according to the amount and distribution of EVs, charging pile location, and availability information etc. [1]. Smart grid cannot only intelligently deliver electricity but also manage 
power facilities taking advantage of real-time information exchanging, routing, and sharing between providers and consumers [2]. Smart grid helps exchange supply and demand information to achieve balanced and smooth operation of the system. Inappropriate charging plan makes charging procedure more difficult and time consuming. On the other hand, optimized and wellorganized charging service may reduce traffic jam and extra traffic volume [3].

Dynamic traffic simulation models are frequently used to support decision-making on charging. Mobility modeling and simulation of EV charging are useful in analyzing how to deploy the charging piles in certain area and how EVs find the best charging pile in that area. Such models and simulation methods are necessary essentially because the real world data about EV use is scarce due to the low adoption levels to date [3]. In the Internet of Vehicle (IoV), interconnected vehicles can share information about the charging experience and traffic condition to help achieving better charging plan. Different charging scheduling algorithm and deployment of charging service can be verified and optimized according to the simulation result. With the aid of simulation where the simulated mobile EV charges and discharges as the real entities, more and more innovative design of IoV can be verified and optimized with low cost and high trustworthiness before they are deployed in the real world.

Many research activities are visible on mobility and scheduling of EVs and the optimal deployment and arrangement of charging piles to evaluate and enhance the designs of such an EV-induced smart grid. EVs and charging pile have mutual impact on each other. A wellorganized smart grid helps to increase the efficiency of grid and enhances the experience of travelers.

Some work regarding the EV charging are focused on the supply side, such as transmission level or distribution level. Authors in [4] considered case studies with different EV charging patterns and estimated the maximum number of EVs that a distribution network can accommodate based on an $\mathrm{N}-1$ contingency condition. With advanced sensing and control technologies, the development of demand response charging can be more creative and flexible with many more possible options. In [5], coordinated charging distribution level is proposed to minimize the power losses and to maximize the main grid load factor.

Some studies focus on the demand side in terms of the impact of the behavior of EVs on the charging efficiency and utility. The mobility of vehicle may pose radical impact on the energy consumption of EVs and pattern of load of charging pile at the supply side of the grid. A smart grid should be adapted to the complex and dynamic distribution and density of EVs. What's more, a smart grid should support and facilitate the EVs with more effective and economical traveling plans. There are lots of works on the mobility simulation of EVs. Seine Aval Véhicule Électrique (SAVE) is France's biggest trial program of all-electric mobility, ultimately involving 100 EVs and 150 charging piles [6]. The MATSim [7] is an agent-based travel demand simulation framework to simulate large-scale traffic scenarios. In [8], the authors proposed an energy consumption models and a computing method for different mobility modes are put forward to calculate the energy consumption, based on discussion of the influence factors of energy consumption under different driving mode, for example is acceleration, constant speed, deceleration, and idle speed. The SAE J1772 [9] series define the standard for electric connectors and their charging system architecture, including physical, electrical, communication protocol, and performance requirement.

In this paper, we develop a simulation platform to model the entities of smart grid such as mobile EVs and charging piles. The key properties and behaviors that are related to the mobility and energy exchange process are abstracted and mirrored to the simulated EVs, and the digital entities may move and charge just as the twin of the real-world EVs. By introducing a mobility model with digital twin, we simulate their behaviors and interactions to study the efficiency and quality of the charging pile and EVs from both supply side and demand side. The simulation platform can help optimize the charging scheduling and navigation algorithm. It also provides support for planning of locations of charging piles and strategies of commercial operational services.

In the flowing sections, we construct the models of EVs and charging piles according to different profiles and configurations. Then we introduce models of different transportation behaviors such as destination choices and route choices in daily routine to simulate the scenarios in day-to-day traffic conditions. By simulating over a certain period of time in a given area where a large-scale fleet of EVs is scattered and charging facilities are equipped.

\section{Methods of mobility modeling}

In this paper, we introduce the EV model with digital twin to simulate the realistic EV in the real world. The digital twin EV is modeled with the same physical and electrical profiles and traffic characteristic as the correspondent EV in terms of mobility pattern, travel demand, energy consumption, and supply. The movement of digital twin EV is fine controlled by a series of driving operations such as steering, accelerating, and braking to impose direct control to the movement of EVs. The digital twin EVs consume exactly the same energy as the real-world EVs do according to energy 
consumption model based on electrical profile of EV. The digital twin EV is also scheduled according to randomized travel agenda and different charging plan to simulate the time-varying travel intent and charging activities of EV drivers.

By using this method, we can simulate the realistic and reasonable moving trajectory of vehicle and can synthesize smooth trajectories such as all kinds of possible curves instead of zigzag arbitrary traces as most other mobility models do. Via analysis of experiment data collected by the simulation such as driving distance, charging frequency, charging cost, waiting time, travel times, average charging speeds, queue lengths, departure, and arrival patterns, we can evaluate the efficiency of a charging policy on both EV and charging pile sides in different scenarios.

\subsection{Mobility modeling of digital twin EV}

It is necessary to establish realistic mobility models of EVs as if they are in original moving environment. The digital twin EVs, thus, are modeled as what they are in real world. The EV mobility model is composed of three main components: modules of energy consumption, motion synthesis, and travel agenda. The energy consumption model simulates the process of charging and discharging according to the driving mileage and charging schedule. The motion synthesis model simulates the driving process of EVs by updating the time series in different geo-locations and coordinates of EVs to get realistic trajectory and mileage. The travel agenda model is used to simulate the diaries of drivers to impose traffic load and charging demand to the system. The motion synthesis model can navigate EVs to different interesting sites assigned by agenda model, and the energy consumption model maintains and monitors the status of charging (SOC) of EV batteries to make the charging choice. The key of digital twin design is to simulate its real-world counterpart precisely.

\section{Energy consumption model}

An EV has specific electrical and physical profile such as battery size, available energy, Miles Per Gallon equivalent (MPGe), charging power, and speed. In the simulation scenarios, to make the digital twin EV consume the equivalent energy as the real EV, we introduce the energy consumption module to the EV mobility model. We record the length of trajectory of EVs and estimate the energy consumption to update the SOC of EV batteries. Leveraging dynamic simulation of EV discharging process, the EVs can make charging decision to prevent the battery from being depleted completely.

To calculate the energy consumption, the energy efficiency and power of EVs should be quantified according to the profile. For fuel vehicles, the energy cost is evaluated in terms of fuel consumption within certain diving mileage. The calculation of fuel energy unit per miles is common in Europe and Asia to reflect the power used by EVs, and it is expressed in the metric system as liters per $100 \mathrm{~km}$. Instead of energy unit per miles, miles per unit of energy such as MPG is used in America. According to the US Environmental Protection Agency, passenger vehicles sold in the USA had the highest fuel economy rating of 23.8 MPG. As 1 mile is equal to 1.609 kilometers $(\mathrm{km})$ and 1 gallon is equal to $3.785 \mathrm{l}$, so 23.8 MPG means 10 kilometer per liter, or 10 liters per 100 kilometer.

For EVs, the MPGe figure reflects the energy efficiency, and it is how the Environmental Protection Agency (EPA) converts the figure into a term that's correspondent to MPG used by fuel vehicles. Labels on EVs often show their all-electric fuel economy in the MPGe format. For example, the Prius is rated at 95 MPGe; the Accord is rated at 115 MPGe. However, consumers are probably most accustomed to buying electric energy from charging pile in kilowatt-hours (kWh). $\mathrm{kWh} / 100$ kilometer is a commonly used measure of efficiency and the most important operating-cost figure for simulation of EV battery. So we should convert the MPGe to kWh/100 kilometer [3, 6].

This calculation is based on the EPA's formula of $33.7 \mathrm{kWh}$ being equivalent to 1 gallon gasoline energy. The EPA uses an established energy standard of 115,000 BTUs (Heat is measured in British Thermal Units) per gallon of unleaded gasoline, where $1 \mathrm{BTU}$ is about $1055 \mathrm{~J}$. Since $1 \mathrm{~J}$ is $1 \mathrm{~W} \mathrm{~s}, 1 \mathrm{kWh}$ is $3.6 \mathrm{MJ}$. Then $1 \mathrm{kWh}$ is equal to $3.6 / 1055=3412.32 \mathrm{BTU}$ [1, $3,6]$. To create the same amount of heat which 1 gallon of gasoline generates, $115,000 / 3412.32=33.701$ kWh of electricity is needed equivalently. For example, the 2014 Ford Focus Electric with an EPA combined fuel efficiency rating of 105 MPGe is driven on a combined (city/highway) MPG cycle, it requires $100 / 105 \times 33.7=32.09 \mathrm{kWh}$ of electricity to travel 100 miles $(32 \mathrm{kWh} / 100$ Mile or $320 \mathrm{Wh} /$ Mile). According to this formula, we can calculate the battery usage dynamically when EVs are driving in the simulation scenarios. Table 1 shows the converted MPGe figures of EVs to Wh/Mile which is used to calculate the energy consumption in simulation.

Based on the energy calculation, energy consumption model of EVs can deduct correspondent energy cost from the total budget of batteries according to the mileage of the journey in the simulation. This energy consumption model only shows the average level of the EVs, and the energy cost for given mileage may vary dramatically due to the behavior of EVs such as acceleration and condition of the road. 
Table 1 Covert labeled MPGe of EVs to Wh/Mile

\begin{tabular}{|c|c|c|c|c|c|c|}
\hline Vehicle Brand & kWh/100 Miles & Wh/Mile & Gallon/100 km & km/liter & Liters/100 km & MPGe \\
\hline 2008 Ford F150 truck5e & 240.72 & 2407.24 & 4.44 & 5.96 & 16.78 & 14 \\
\hline Fuel Cell Vehicle & 96.29 & 962.90 & 1.78 & 14.90 & 6.71 & 35 \\
\hline Focus EV & 32.10 & 320.97 & 0.59 & 44.69 & 2.24 & 105 \\
\hline 2015 Tesla Model S 70D & 30.64 & 306.38 & 0.57 & 46.82 & 2.14 & 110 \\
\hline Nissan Leaf & 30.36 & 303.62 & 0.56 & 47.25 & 2.12 & 111 \\
\hline Plug-in Prius in EV mode & 23.90 & 239.02 & 0.44 & 60.02 & 1.67 & 141 \\
\hline
\end{tabular}

\section{Travel agenda model}

The characteristic mobility and related synthesized trajectories of a batch of EVs may pose great impact on the energy consumption and charging demand, and then are key to the trustworthiness of the EV charging simulation. Moreover, in order to make the journey of EVs more realistic, we introduce agenda model to the mobility model based on public daily traffic survey data. The events in the agenda are generated on a random basis in time and space, described as event start time, deadline, dwell time, queuing time, service time, leaving time, patronage frequency, service type, location, accommodating population, priority, and profits, etc. For example, an EV driver may go to a small restaurant near working place at 11:00-12:00 for 2-4 times every week with medium priority and has lunch there for about $40 \mathrm{~min}$ after $10 \mathrm{~min}$ of ordering and waiting. Event generator inserts a task item into the EV's agenda list with randomly varied properties based on the event description. The EVs execute the task according to the current status of task load and energy budget. If the EVs are available at that time without other higher priority tasks such as charging and repairing to handle, it navigates there and detains for certain time before it picks up the next task to execute. In this event-driven process, the EVs create time series behaviors and generate continuous and realistic trajectory as a EV may behave in the real world.

To reflect the variety of EVs' behavior, we define six types of agenda templates to reflect different typical travel patterns such as travel patterns of routine prone, shopping prone, weekend prone, night shift prone, busy prone, and lazy prone. All the modeled EVs have presumed agenda to follow and drive to a series of points of interest in the simulation while satisfying the energy budget constraint by periodical charging. The mobility and the charging schedule of EVs impose correspondent impact to the daily traffic and charging demand on the charging piles. By simulating traffic and charging load in a large scale of interconnected EVs, we can evaluate the performance of different proposed algorithms of charging scheduling and charging pile deployments.

2. Motion synthesis model
In this paper, we introduce a fine controlled motion synthesis method to simulate the moving process of EVs. We model the EV's motion as how EVs are controlled and operated by a driver on moving $[8,10]$. The motion synthesis model abstracts the primitive motions of EVs as time series behavior such as steering, accelerating, and braking. This microscopic mobility model delivers a convincing trajectory with more practical and accurate mileage and energy consumption. Each EV is modeled as visual object with physical and geometric properties such as vehicle body size, current moving direction, and orientation (the orientation of the head of vehicle). Each $E V$ is assigned with operation and motion parameters such as steer angel and range, speed, and acceleration for the monitoring of EV safety [11, 12]. The mobility of the dynamic moving is controlled by basic operations of EVs such as steering, braking, and accelerating. The time varied position of an EV is expressed as function of these operation parameters.

In the motion synthesis model, to get accurate trajectory of EVs, the space coordination system should be mapped between device screen and the real field of scenario [13-15]. The time coordination system should be mapped between computer system time and the time of scenario to be simulated. Let $\lambda$ be the space scale and $\tau$ be the time scale. $\lambda$ denotes the amount of scenario miles that are mapped to a computer screen pixel, and with the unit of mile/pixel. $\tau$ denotes the amount of scenario hours that are mapped to a computer millisecond, and with the unit of $\mathrm{h} / \mathrm{ms}$. Then the speed of EVs can be denoted as moving speed of EVs on screen. The vehicle velocity $v$ in unit of mile/h can be mapped to screen speed $s$ in unit pixel/s with a transmission ratio of $\mathrm{\tau} / \lambda$. We have $s=\nu \cdot \tau / \lambda$ [16]. For example, given $\tau=$ 0.001 ( 1 computer second for $1 \mathrm{~h}$ in simulated scenario), and $\lambda=0.3$ ( 1 computer pixel for 0.3 mile in simulated scenario), speed of $30 \mathrm{mile} / \mathrm{h}$ can be mapped to screen speed of $30 \times \mathrm{\tau} / \lambda=10 \mathrm{pixel} / \mathrm{s}$.

To simulate the moving process of EVs, the dynamic position and direction of EVs should be calculated according to the mobility parameter and the EV profile [17]. Given the position of $\mathrm{EV}$ at time $t$ is $(x, y)$, the position and direction of $\mathrm{EV}$ at $t+\Delta t$ ( $\Delta t$ is the interval slot of refreshing) can be obtained by a parametric equation as follows [18]: 


$$
\left\{\begin{array}{r}
x^{\prime}=x+v \cdot \frac{\tau}{\lambda} \cdot \Delta t \cdot \cos \left(\theta \cdot \frac{\pi}{180}\right) \\
y^{\prime}=x+v \cdot \frac{\tau}{\lambda} \cdot \Delta t \cdot \sin \left(\theta \cdot \frac{\pi}{180}\right)
\end{array}\right.
$$

where $x^{\prime}$ and $y^{\prime}$ is the coordinate of EV after next refresh time slot given the current position $(x, y)$.

As to the vehicle direction and orientation angle $\theta$ in degree, it also varies as moving with certain vehicle wheel steer angle $\alpha$ in degree. Let $\theta^{\prime}$ be the vehicle direction angle at time at $t+\Delta t$.

If the vehicle moves with fixed wheel steer angle $\alpha$, the vehicle of cause moves in a circle with a steering radius $R$. Let vehicle body length be $L$, if the steer angle $\alpha$ is 0 , the vehicle drives straight and the $R$ is $\infty$, and if the $\alpha$ is $\pm \pi / 2$ or $\pm 90^{\circ}$, the front wheels are vertical to the vehicle body, the vehicle drives in a circle with $R$ equal to $L$. Assuming the angular velocity (in radian) is $\omega$, and $\alpha$ is kept the same during $\Delta t$, the change of vehicle orientation after $\Delta t$ is correspondent to the arc angle between the point $(x, y)$ and $\left(x^{\prime}, y^{\prime}\right)$, i.e. $\Delta \theta=\omega \Delta \mathrm{t} \frac{180}{\pi}$ in degree. We have $\theta^{\prime}=\theta+\omega \Delta \mathrm{t} \frac{180}{\pi}$

As $\omega=\frac{S}{R}$, where steering radius $R$ is related to the vehicle body length and vehicle wheel steer angle $\alpha$. Then we have $\theta^{\prime}=\theta+v \cdot \frac{\tau}{R \lambda} \Delta t \frac{180}{\pi}$.

So we can present the radius as $R=L / \sin \left(\alpha \cdot \frac{\pi}{180}\right)$, and we have:

$$
\theta^{\prime}=\theta+v \cdot \frac{\tau}{\lambda} \cdot \Delta t \cdot \sin \left(\alpha \cdot \frac{\pi}{180}\right) \cdot \frac{180}{\pi}
$$

The velocity of the EV is also time-varying and the vehicle velocity $v$ can be calculated as:

$$
v^{\prime}=v+\Delta t \cdot A
$$

where $A$ is the acceleration of EV.

During moving, the EVs adjust the steer wheel and change acceleration dynamically, the mobility model can calculate and update the time-varying position and orientation of vehicle according to Eqs. (1), (2), (3). An EV then can move from current position to a new position on screen at a fine updating interval and generate smooth and realistic trajectory. The time series behavior of an EV is the basis of traffic generation in the simulation of a large-scale charging scenario. Due to the mathematical simplicity of the approach and the fact that relatively few control parameters are required, the motion synthesis model is easy to be used in simulation platform and can achieve high performance especially in scenarios with large scale of EVs [19].

In the simulation, when an EV is assigned to a task according to the travel agenda model, the EV is navigated automatically to the destination related to the task. A direct and straight way to approaching to the target is U-Turn algorithm. In this algorithm, when EV is assigned to a new target, it adjusts its orientation to the direction pointing to the target. The adjustment of orientation can be achieved by resetting the orientation angle forcedly, or by adjusting the previous angle to the final angle pointing to the targets bit by bit by steering the wheel with certain angle. Reset of the orientation is simple to realize, but the trajectory of EV shows as a straight line from the current location to the designation [20-22].

The straight trajectory is not realistic and may cause inaccurate mileage. So we choose to adjust the orientation angle by steering the wheel with smooth operation. We calculate the difference between the current orientation angle and the correct orientation angle that is aiming to the target. Then EVs steer to the right or left by a fine wheel angle bit by bit with corresponding adjustment value, and EVs keep steering for a certain amount of time, and EVs arrive at a new position with updated orientation angle which can be obtained according to the Eq. (2). The EVs repeat this procedure until they aim to the right orientation and approaches the destination.

Figure 1a shows the synthesized trajectories of a batch of EVs evacuating from the same source site to different destinations by the proposed mobility model. Instead of just adjusting the EV direction to the target and drive straight to the target, we use more realistic and less radical navigation method. In Fig.1b, the mobility model is used to simulate the traffic flow in a scenario of signalized flat intersection. The EVs follow the respective lanes of the road, by adjusting the angle smoothly, digital twin EV can keep realistic trajectory during marching in queue as how the real EV does.

The route choice model used in the navigation is gravity-based attraction algorithm, where the EVs steer the wheel smoothly and approach the target gradually [23]. The trajectories of EVs are more smooth and then more realistic compared with other mobility models. As to the navigation algorithms, the EV may choose the path to approaching the target or destination according to different matrices and strategies.

\subsection{Modeling of charging pile}

Accompanying to the digital twin modeling of discharging process of EVs, the digital twin of the charging pile is also needed to simulate the charging process of EV. The digital twin of charging pile is modeled with a certain number of plug-in electric connectors with different geographical, physical, and electrical parameters, such as GPS location, type of plugs, charging voltage, current, temperature, price, and power.

The energy exchanging conducts according to the constraints of the BMS (Battery Management System) and charging profiles of EVs and piles. What's more, the quality of a charging service such as charging speed, 


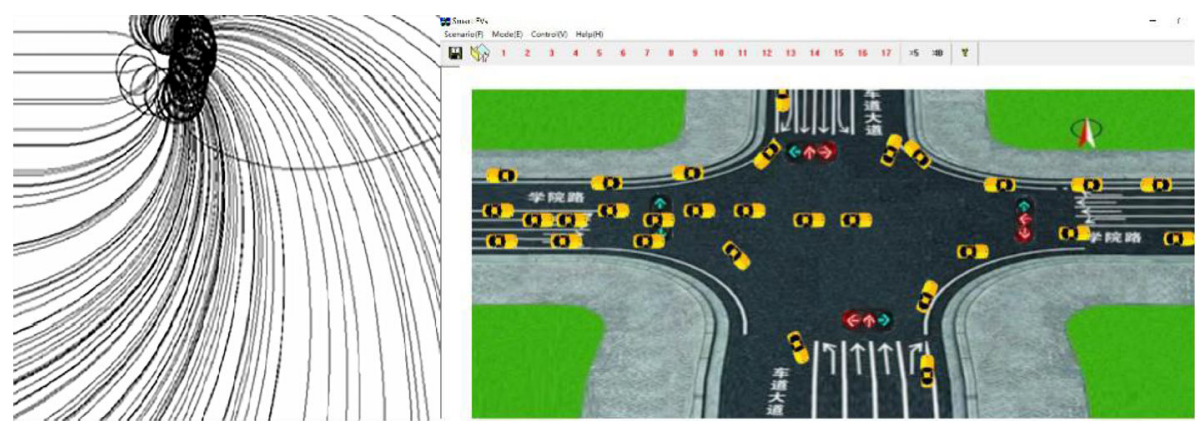

(a) Trajectory of evacuation

(b) Simulated traffics at an signalized intersection

Fig. 1 Synthesized trajectories of EV. a Trajectory of evacuation. b Simulated traffics at an signalized intersection

number of plugs, capacity of parking lot, availability of pile, waiting time, waiting queue line, and charging frequency may vary dynamically and should be considered when EVs make charging plan. With the communication capacity provided by the smart grid and VANET, the charging pile can negotiate with an EV based on these running data. It can also sense the distribution of nearby EVs and their charging demands [24-26]. Charging pile may advertise the quality and availability of service to the EVs which can make decision of charging choice to eliminate the waste of time and energy in case of choosing another pile due to capacity limit and profile mismatch. When an EV chooses to drive to the charging pile, the charging scheduler may prepare the preserved matched pile and correspondent power load budget according to the physical and electrical profile of the given vehicle type [27]. The request reserved renegotiation procedure eliminates possibility of the denies of service for the incoming request due to limited resource such as power and related service infrastructure.

\subsection{Route choice model in task processing}

Each EV is assigned to an agenda template to simulate travel demand patterns according to the travel agenda model. We select 17 types of most typical interesting sites (such as office, park yard, bank, medical center, school, and restaurant) to construct the events pool according to spatially distributed travel demand $[2,28]$. The event site is randomly dropped on the map at

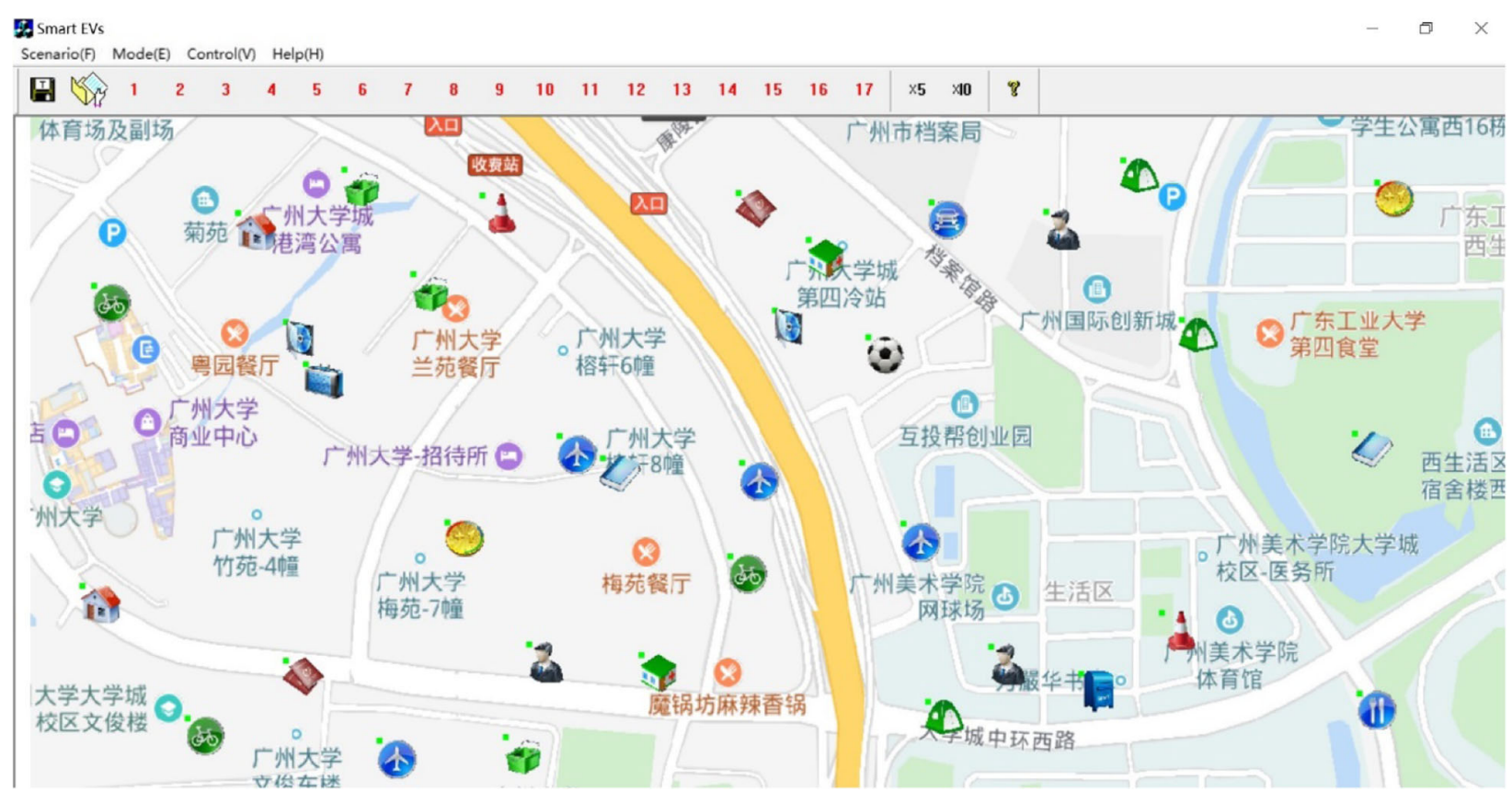

Fig. 2 Time series data based events map 
specific event occurrence intensity. The density and distribution of the events can be configured to reflect the difference in commercial and administrative prosperity of different areas and sections in the simulation scenarios.

The events or tasks can be seen as dynamically created time series data with time and special property to simulate the time-varying travel demands of EVs (Fig. 2). When EVs approach the event site assigned by the agenda, EVs may dwell there for certain period of time to simulate the parking or charging activities before driving to the next task. When the task is processed, bonus is scored by the EVs to calculate the activity intensity of EVs. EV repeats this task routine and generates trajectory according to the motion synthesis model. Energy consumption and supply model is responsible for updating the SOC of EVs and route choice model in charging makes charging decision during the task processing. The route choice model in task processing imposes simulated traffic flow to the scenarios and generates the charging demand by the travel mileage of EVs when it drives to different interesting sites according to the travel agenda model.

\subsection{Route choice model in charging}

Within route choice model in charging, we have three options. Firstly, we use the static pre-trip route choice models, where EVs are assumed to choose their route (from origin to destination) before departure according to information obtained or past experiences and do not switch routes or destination (target charging pile) while driving for charging. For in-route route choice models, travelers observe prevailing traffic conditions and quality of charging services as they travel, and make route choice decisions accordingly. EV may change their target charging pile during the trip adaptively [29]. In hybrid route choice models, travelers rely on both on pre-trip route decisions and in-trip decisions. They may find a better choice according to different conditions. Some travelers may decide to switch to an alternative route when receiving advertised guiding information from the smart grid.

In the simulation, we presume that all the EV initiate with a fixed energy level that is much higher than the critical level (with enough energy to drive to the nearest charging pile). A proper charging plan is important to help overcome range anxiety of EV driver, and avoid an EV battery running out of energy before a destination is reached. The charging scheduling model should confirm this safe energy status and make prompt and proper charging decisions. Our route choice model for charging considers three basic requirements to simulate this process of charging of EVs. EVs should decide when, where to charge, how long to charge, and where to go after charging.
As to the time point to toggle the route choice, there are several options. An EV may choose to go to charging pile whenever the SOC of EV approaches certain fixed threshold value which should be higher than the critical level. EV may also choose to charge whenever it approaches some charging pile which is within a close distance range (i.e., 1-2 miles) from the current location. Some EVs choose to charge on a regular time basis or choose to charge according to the random intend of EV drivers.

When an EV decides to go to charge, the next decision for the EV is where or which charging pile to choose. Distance, type of charging pile, service quality, and availability of pile should be taken into account respectively or associatively. Take short-distance-first policy as an example, the model can choose the nearest pile from the current location of an EV, or it may not go to charge right now but choose the pile which is nearest to the upcoming trajectory along the way to the next destinations according to the travel agenda model [30]. When certain charging pile is set as the target, the motion synthesis model navigates the EV to the destination.

The EV then decides how much energy to supply and how long or how much the charging process takes. The time and amount may vary due to the constraints such as time and budget limit, capacity, and policy of parking lots [31]. To simulate this process, the model provides 4 basic options for EVs. The most conservative option is to charge the minimal amount to keep the SOC above the critical level. EV may also choose to charge for a fixed amount of energy to simulate the regular consumption custom of EV driver. Some EV may choose to let the battery fully charged or to a certain fixed threshold. Route choice model demonstrates the variety in charging behavior of EVs in simulation.

When EV finishes charging, it may decide where to go after interruption of charging. Some policy may choose to return to the interrupted point where the EV detours to the charging pile while processing the daily tasks. EV may also not go back but continue to navigate to the next event site based on the current charging location. Different return policies may have different impact on the performance and efficiency of charging.

These multimodal decision-making policy of digital twin EV provides high freedom of motion in the charging process of EV and helps simulate the complex possible mobility pattern of EV in real world. The fine controlled digital twin EV model can increase the trustworthiness of the motion simulation of IoV.

\section{Experiments}

To validate the feasibility and effectiveness of the proposed EV mobility model, and show how EV mobility affects the traffic system and the energy system, we set up 
a simulation scenario of a $3 \mathrm{~km} \times 6 \mathrm{~km}$ campus area in Guangdong Province as the background with 500 digital twin EV nodes scatted randomly and 280 plugs of digital twin charging piles deployed. Each EV has been assigned to random initial configuration of parameters such as battery capacity, MPGe, maximum speed, and charging power $[18,32]$. A digital twin EV is automatically navigated according to the agenda tables and charging scheduling algorithms. Figure 3 shows the simulation scenario.

The utility and efficiency of charging pile can be analyzed according to the recorded statistical simulation data such as charging frequency and waiting time of EVs. We find a manifest asymmetry load pattern of different pile due to the location and mobility of EVs. Based on this variety, statistical analysis can be conducted by introducing different traffic flows of EV and travel tasks to simulate different application scenarios. The mobility and charging process of a large scale of EVs is simulated in the investigated area.

\section{Results and discussion}

To evaluate the performance of different charging scheduling algorithms, several factors are set to analyze the efficiency of the charging. The detour distance of an EV is defined as the difference between the total mileage of journey and the total one without charging activities. In a given simulation time, the total detour distance of all EVs is calculated and used as a key factor to evaluate the performance of charging scheduling algorithms and the feasibility of pile arrangement. Considering that the EV may change its task execution sequence and choose different route after it finishes charging, the route of EV may be completely different with the route of task processing without need of charging. The detour distance may be difficult to calculate. To eliminate the interference of detouring on the travel plan and route during task processing, we introduce a reference digital twin node for each digital twin EV. The reference EV has a battery with infinite capacity without charging demand, while it has the same configuration and agenda template with its correspondent digital twin EV. The only difference of reference $\mathrm{EV}$ and the digital twin $\mathrm{EV}$ is that the reference EV does not need charging and the energy cost is only related to task processing activities. All the EVs compete with each other in the same traffic conditions and the same event density; we evaluate the digital EVs by analyzing the difference in the energy cost of charging activity while complete the given work load. The mileage of reference EVs is only related to the task processing without charging, and then the detour distance can be easily calculated as the mileage difference between $\mathrm{EV}$ and its reference EV.

To evaluate the feasibility of pile arrangement, we use another factor to validate the utility of charging piles. Each pile is assigned a service coverage range, whenever an EV enters the coverage area of a charging pile, a contactcounter is increased by one. The contact-counter of pile reflects the intensity of traffic flow passing by and can be used as a factor to evaluate the importance of a pile in a charging service grid. The higher the contact-counter, the pile has higher possibility to provide charging service to the EVs. The ratio between the investigated charging times and the contact-counter of a pile reflects the potential of the pile. If charging scheduling algorithm is subtly designed, the ratio can be enhanced and better utility of

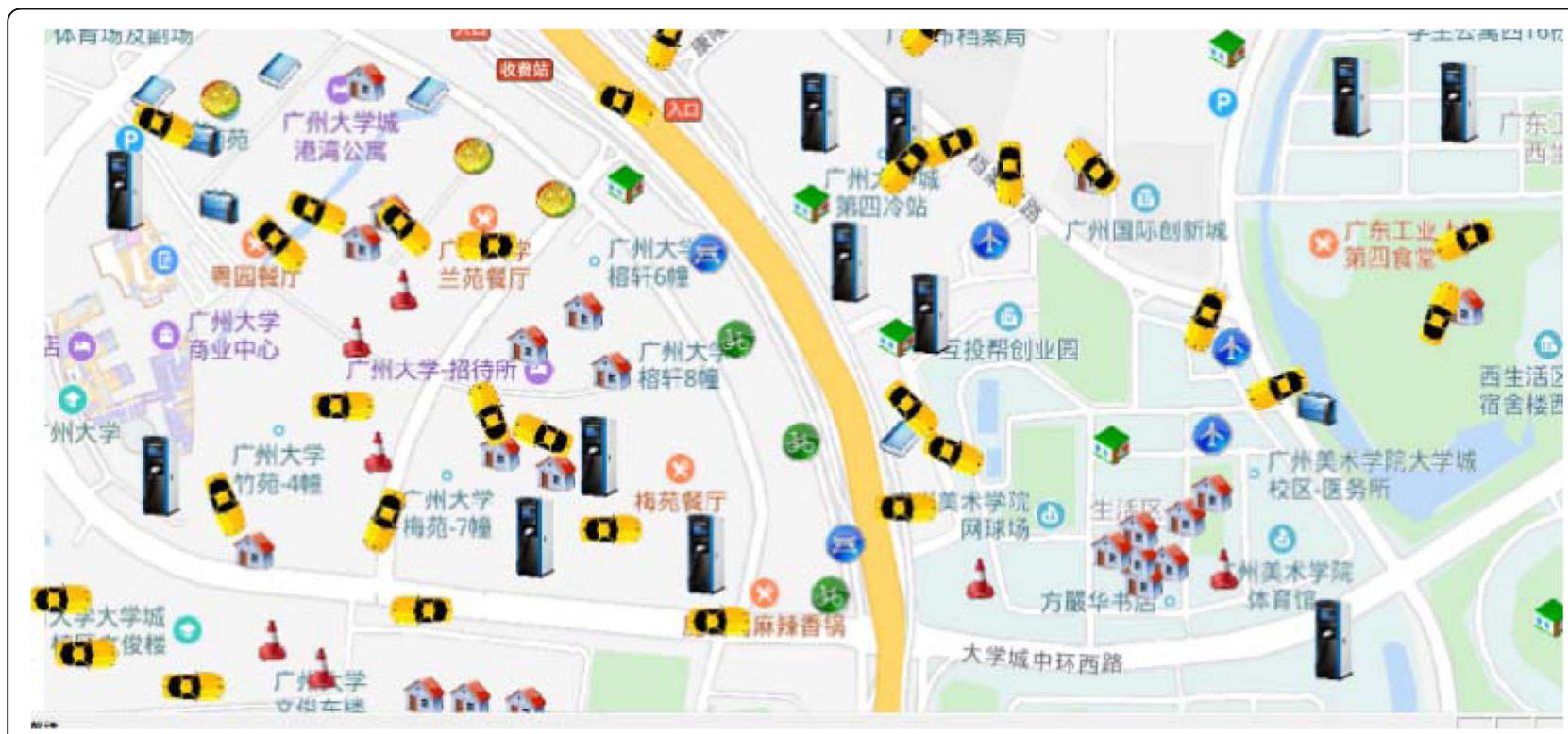

Fig. 3 Simulation scenario of network of EVs 


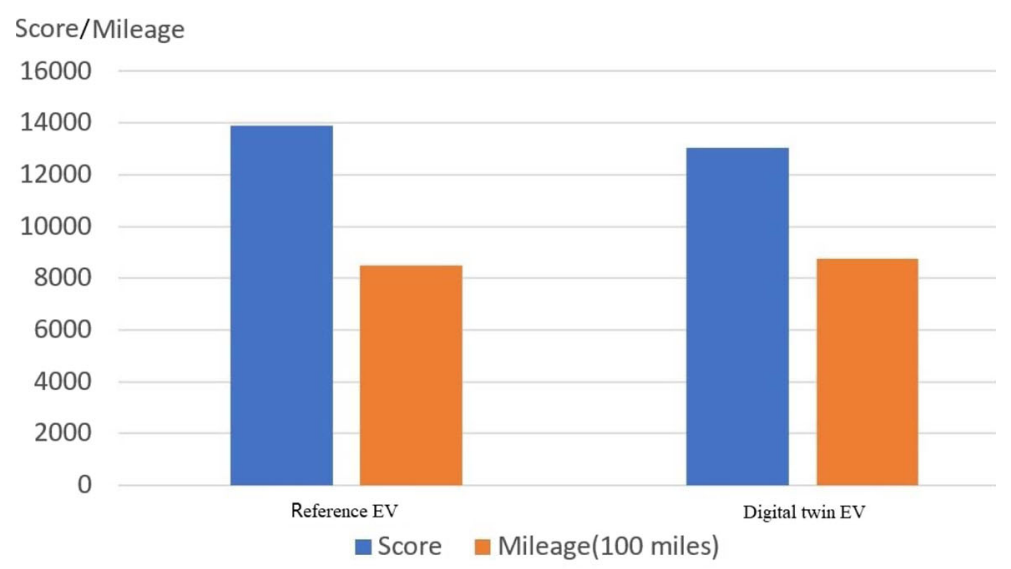

(a). Scenario1 with 1 pile

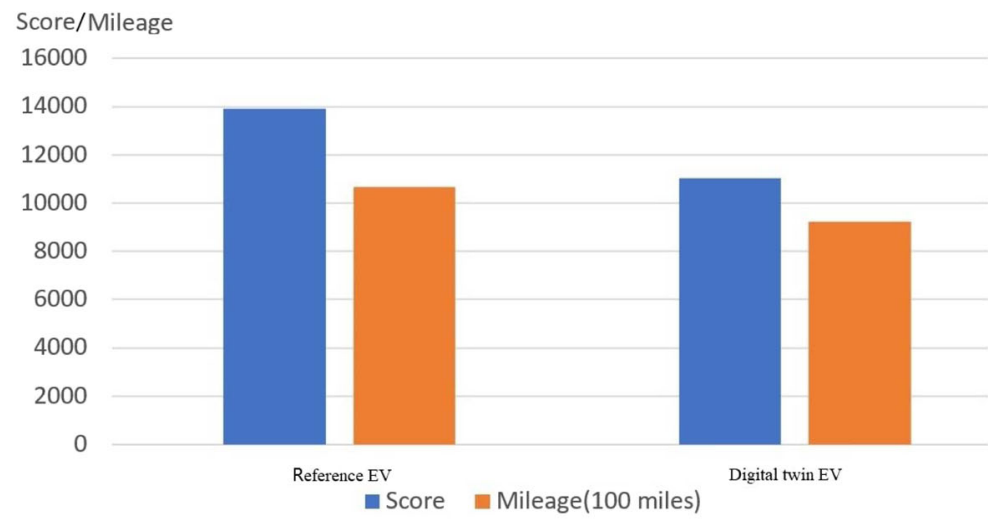

(b). Scenario 2 with 4 piles

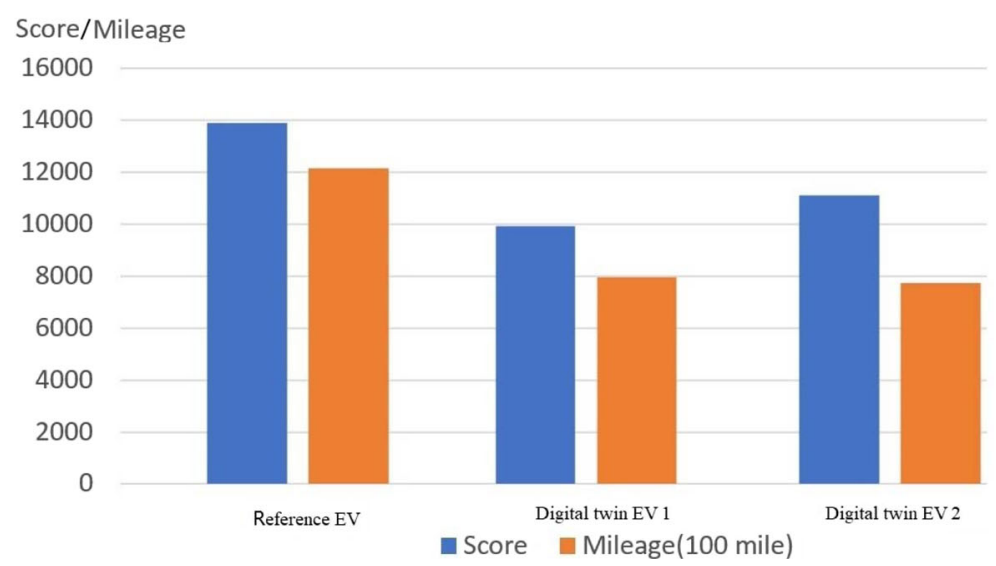

(c). Scenario 3 with 8 piles and 3 EVs

Fig. 4 Score and mileage of reference EV and digital EVs in 3 scenarios. a Scenario 1 with 1 pile. b Scenario 2 with 4 piles. c Scenario 3 with 8 piles and $3 \mathrm{EVS}$

the charging infrastructure can be achieved. On the other side, rearrangement can be made on the deployment of the charging piles according to the simulation results to achieve the final balance among the piles and enhance the utilization of energy grid.
In the simulation, the digital twin EVs and charging pile are modeled as how real-world EVs and piles work in terms of physical and electrical characteristics. The energy consumption and charging process are simulated by energy model on the EV and charging pile side. The 
mobility and behaviors of EVs are motivated by scheduling their order taking and order processing according to the given agenda model. We compare the mileage of digital twin EVs and its reference EV. The EVs run in the same scenario with the same traffic environment and the events density is set to be proportional to the number of EVs to keep the equal task load for each EV. The digital twin EVs can choose charging pile from a set of charging piles. Different amount and arrangement of charging piles is set to study the impact of charging pile deployment on the charging efficiency. After certain simulation period, we calculate the mileage and the task score of the two compared node. We set three scenarios to show how to use the simulation platform to evaluate the feasibility of pile arrangement.

In the first scenario, we provide only 1 charging pile located at the center of the map. In the second and third scenario, we provide 4 and 8 charging piles scattered on the map with uniform distribution respectively. In the 3 scenarios, digital twin EVs and reference EVs navigate to the randomly generated event sites to earn task score in a competitive manner. The task score reflects the work load and the traffic intensity. The digital twin EVs and reference EVs are homogeneous configured and share the same background in terms of traffic and task load, and the only difference is the arrangement of the charging piles. To make these three scenarios comparable in principle, we use the work load of a common reference EVs as indicator, instead of using the simulation time. All the simulation run until the reference EV scores the same value. In the simulations, we collect data of twin EVs in 3 scenarios where the reference EV earns the same amount of task score (13,900 points). We find that the digital twin EV in the first scenario scored less compared with their competitive reference EV due to overhead of charging in terms of time and mileage. The reference EV takes more orders while the digital twin $\mathrm{EV}$ is engaged in charging. The efficiency ratio (ratio of the earned score to mileage) is $1.6 \%$ and $1.0 \%$ for reference EV and digital twin EV.

In the second scenario, when the reference EV scores the same as in the first scenario, the digital twin EV scores 11,030 with ratio of $1.3 \%$ which are much higher than it does in the first scenario. The difference between the reference node and digital twin node in ratio is decreased to $0.1 \%$. The enhancement in efficiency is because that the digital twin EV has more options for charging and saves detour mileage and time. The decrease of ratio of reference indicates that the digital twin $\mathrm{EV}$ is more competitive due to decreased charging overhead.

In the third scenario, we create 50 digital twin EVs and 1 reference EV (indexed from 1 to 51) with 8 charging pile scattered. The first digital twin EV (node 1) is set to choose the unique charging pile and the others can choose any convenient pile from the 8 candidates. The first digital twin EV scored less compared with their competitive reference EVs (node 51) and the digital twin EV 2 (average of node 2 to 51) as well due to more detour distance. The digital twin EV 2 also earns less score compared with the reference EV, but achieves comparatively higher efficiency ratio (the $95 \%$ confidence interval for this measurement is $1.44 \pm 0.31 \%$ ) due to convenience of charging. As shown in Fig. 4, simulation results show how the number of charging piles and car-to-pile ratio affects the efficiency of charging, validates the design of EV mobility model with digital twin, and can help evaluate the feasibility of charging plan and deployment of charging piles.

\section{Conclusion}

In this paper, a simulation platform with digital twinbased modeling is proposed to conduct replication of charging and discharging process of large-scale mobile EVs in different kinds of dynamic scenarios. To achieve the realistic mobility of $\mathrm{EV}$, we introduce the fine operational motion parameters such as steer angle, orientation angle, and acceleration to the mobility model to simulate the realistic trajectory when EV is targeting to the targets. A new tracking method of the dynamic position and orientation is proposed to synthesize the reasonable trajectory of EV in day-to-day traffic condition. The key elements and activities are modeled as what they are in real world. The digital twin EVs and charging piles behave and interact with each other just as the real entities, and then the simulation result can be used to evaluate schemes of the deployment and management of EVs and charging infrastructures. Simulations validate the correctness and effectiveness of the proposed platform and the EV mobility digital twin model. The experiments also show the charging efficiency differences against various deployment of charging piles. In conclusion, the proposed platform and model are effective in evaluating EV charging scheduling algorithms and charging position deployments. In the further work, different navigation and charging scheduling algorithm can be introduced to the proposed simulation platform to verify the design of deployment of charging infrastructure and related impact on smart grid [33].

\section{Abbreviation \\ BMS: Battery management system; BTU: British Thermal Units; EPA: Environmental Protection Agency; EV: Electronic vehicle; GHG: Greenhouse gas; loV: Internet of Vehicle; MPGe: Miles per gallon equivalent; SAVE: Seine Aval Véhicule Électrique}

\section{Acknowledgements}

This work is supported in part by the Guangdong Province Key Research and Development Plan (No. 2019B010137004), the National Natural Science Foundation of China (U1636215, 61871140, 61572153, 61572492, 61672020), the National Key research and Development Plan (Grant No.

2018YFB0803504), the Beijing Municipal Natural Science Fundation (No. 
4172006), and the Guangdong Province Universities and Colleges Pearl River Scholar Funded Scheme (2019).

\section{Authors' contributions}

TZ has drafted and designed the work; XL has made the acquisition and interpretation of data; ZL has made the literature search and analysis of data; FD has made the literature search and analysis of data; YJ gives the idea and has substantively revised the paper. All authors read and approved the final manuscript.

\section{Funding}

They are presented in the "Acknowledgements" section.

\section{Availability of data and materials}

The datasets supporting the conclusions of this article are available in the main paper.

\section{Competing interests}

The authors declare that they have no competing interests.

\section{Author details}

${ }^{1}$ Cyberspace Institute of Advanced Technology, Guangzhou University, Guangzhou 510006, China. ${ }^{2}$ Southern University of Science and Technology, Shenzhen 518055, China. ${ }^{3}$ Cyberspace Security Research Center, Peng Cheng Laboratory, Shenzhen 518000, China.

\section{Received: 11 July 2019 Accepted: 30 October 2019}

Published online: 16 December 2019

\section{References}

1. J. Lopes, F. Soares, P. Almeida, Integration of electric vehicles in the electric power system. Proc IEEE 168-183 (2011)

2. Z. Tian, S. Su, W. Shi, X. Du, M. Guizani, X. Yu, A data-driven model for future Internet route decision modeling. Future Generation Comput Syst 95, $212-$ 220 (2019)

3. Tirez, A.; Luickx, P.; He, X.; Rious, V. Optimal charging schedule of an electric vehicle fleet, 7th International Conference on the European Energy Market (EEM), pp.1-6, 23-25 June 2010.

4. Junghoon Lee, Hye-Jin Kim, Gyung-Leen Park, and Hongbeom Jeon. Genetic algorithm-based charging task scheduler for electric vehicles in smart transportation. Intelligent Information and Database Systems, March, 2012.

5. K. Clement, E. Haesen, J. Driesen, The impact of charging plug-in hybrid electric vehicles on the distribution grid[J]. IEEE Transact Power Systems 25(1), 371-380 (2010)

6. S. Babaei, D. Steen, T.A. Le, O. Carlson and L. Bertling. Effects of plug-in electric vehicles on distribution systems: a real case of Gothenburg. Innovative Smart Grid Technologies Conference Europe (ISGT Europe), IEEE, 11-13 Oct. 2010

7. M. Sanchez and P. Manzoni. A java-based ad hoc networks simulator. In Proc. of the SCS Western Multiconference Web-based Simulation Track, Jan. 1999

8. Wang, Z.-P \& Sun, F.-C \& Lin, C. (2006). Computing method of energy consumption in EV typical driving mode. 26. 69-72.

9. Toepfer, C.: SAE electric vehicle conductive charge coupler, SAE J1772. Society of Automotive Engineers (2009)

10. J. Haerri, M. Fiore, F. Filali, and C. Bonnet. Vanetmobisim: generating realistic mobility patterns for vanets. In ACM Workshop on Vehicular Ad Hoc Networks (VANET 2006), Los Angeles, California, September 2006.

11. Q. Tan, Y. Gao, J. Shi, X. Wang, B. Fang, Z. Tian, Toward a comprehensive insight into the eclipse attacks of tor hidden services. IEEE Internet Things J 6(2), 1584-1593 (April 2019)

12. X. Yu, Z. Tian, J. Qiu, F. Jiang, A data leakage prevention method based on the reduction of confidential and context terms for smart mobile devices. Wireless Commun Mobile Comput https://doi.org/10.1155/2018/5823439

13. Z. Tian, Y Cui, L An, S Su, X Yin, L Yin and X Cui. A real-time correlation of host-level events in cyber range service for smart campus. IEEE Access. vol. 6, pp. 35355-35364, 2018. DOl: https://doi.org/10.1109/ACCESS.2018.2846590.

14. M. Li, Y. Sun, Y. Jiang, Z. Tian, Answering the min-cost quality-aware query on multi-sources in sensor-cloud systems. Sensors (2018). https://doi.org/10. $3390 /$ s18124486
15. W. Han, Z. Tian, Z. Huang, S. Li, Y. Jia. Bidirectional self-adaptive resampling in imbalanced big data learning. Multimedia Tools and Applications. 2018. DOI: https://doi.org/10.1007/s11042-018-6938-9.

16. J. Qiu, Y. Chai, Y. Liu, Z. Gu, S. Li, Z. Tian, Automatic non-taxonomic relation extraction from big data in smart city. IEEE Access 6, 74854-74864 (2018). https://doi.org/10.1109/ACCESS.2018.2881422

17. Y. Wang, Z. Tian, H. Zhang, S. Su, W. Shi, A privacy preserving scheme for nearest neighbor query. Sensors. 18(8), 2440 (2018) https://doi.org/10.3390/ s18082440

18. Chunyuan Kang, Tianle Zhang. Realistic traffic data based mobility modeling and simulation of smart EV. In the 3rd IEEE International Conference on Data Science in Cyberspace (DSC), 2018, pp. 854-857.

19. S. Su, Y. Sun, X. Gao, J. Qiu, Z. Tian, A correlation-change based feature selection method for loT equipment anomaly detection. Appl Sci 9(3), 437 (2019) https://doi.org/10.3390/app9030437

20. Z. Wang, C. Liu, J. Qiu, Z. Tian, C. Xiang, Y. Dong, S. Su. Automatically traceback RDP-based targeted ransomware attacks. Wireless Communications and Mobile Computing. 2018. https://doi.org/10.1155/ 2018/7943586

21. J. Rolink, C. Rehtanz, Large-scale modeling of grid-connected electric vehicles. IEEE Transact Power Delivery 28(2), 894-902 (2013)

22. Z. Tian, C. Luo, J. Qiu, X. Du and M. Guizani. A distributed deep learning system for web attack detection on edge devices. IEEE Transactions on Industrial Informatics. 2019. DOl: https://doi.org/10.1109/TII.2019.2938778.

23. Christoph Aldejohann, Jonas Maasmann, Willi Horenkamp, Fritz Rettberg, Christian Rehtanz. Testing environment for vehicle to grid (V2G) applications for investigating a voltage stability support method. In IEEE Transportation and Electrification Conference and Expo (ITEC), Dearborn 2014

24. Road vehicles - Vehicle to grid communication interface - Part 1: General information and use-case definition, ISO 15118-1, 2013.

25. M. J. Rutherford and V. Yousefzadeh. The impact of electric vehicle battery charging on distribution transformers. In Applied Power Electronics Conference and Exposition (APEC), 2011 Twenty-Sixth Annual IEEE, 2011, pp. 396-400.

26. Z. Tian, W. Shi, Y. Wang, C. Zhu, X. Du, S. Su, Y. Sun, N. Guizani, Real time lateral movement detection based on evidence reasoning network for edge computing environment. IEEE Trans Ind Inform 15(7), 4285-4294 (2019)

27. Z. Tian, M. Li, M. Qiu, Y. Sun, S. Su, Block-DEF: a secure digital evidence framework using blockchain. Inform Sci 491, 151-165 (2019). https://doi. org/10.1016/j.ins.2019.04.011

28. Z. Tian, X. Gao, S. Su, J. Qiu, X. Du, M. Guizani, Evaluating reputation management schemes of Internet of Vehicles based on evolutionary game theory. IEEE Trans Vehicular Technol 68(6), 5971-5980 (2019)

29. Y. Xiao, V. Rayi, B. Sun, X. Du, F. Hu, M. Galloway, A survey of key management schemes in wireless sensor networks. J Comput Commun 30(11-12), 2314-2341 (Sept. 2007)

30. X. Du, Y. Xiao, M. Guizani, and H. H. Chen. An effective key management scheme for heterogeneous sensor networks. Ad Hoc Networks, Elsevier, Vol. 5, Issue 1, pp 24-34, Jan. 2007.

31. Y. Xiao, X. Du, J. Zhang, S. Guizani, Internet protocol television (IPTV): the killer application for the next generation internet. IEEE Commun Mag 45(11), 126-134 (Nov. 2007)

32. X. Du, H.H. Chen, Security in wireless sensor networks. IEEE Wireless Commun Mag 15(4), 60-66 (Aug. 2008)

33. X. Du, M. Guizani, Y. Xiao, H.H. Chen, A routing-driven elliptic curve cryptography based key management scheme for heterogeneous sensor networks. IEEE Trans Wireless Commun 8(3), 1223-1229 (March 2009)

\section{Publisher's Note}

Springer Nature remains neutral with regard to jurisdictional claims in published maps and institutional affiliations. 